\title{
DUAL-LAYER GRID POLARIZERS FOR MM AND SUB-MM WAVES: THEORY AND EXPERIMENT
}

\author{
V.B. Yurchenko ${ }^{1}$, M.L. Gradziel $^{2}$, J.A. Murphy ${ }^{2}$ \\ ${ }^{1}$ Institute of Radiophysics and Electronics, National Academy of Sciences of Ukraine \\ 12 Proskura St., Kharkov, 61085, Ukraine \\ Tel +380577203569 Fax +380573152101 E-mail: v.yurchenko@nuim.ie \\ ${ }^{2}$ Experimental Physics Department, National University of Ireland - Maynooth \\ Maynooth, Co. Kildare, Ireland \\ Tel +35317083771 Fax +35317083313 E-mail: anthony.murphy@nuim.ie
}

This work is concerned with design, manufacturing and testing of enhanced dual-layer grid polarizers for millimeter (MM) and sub-millimeter (sub-MM) electromagnetic waves. Enhanced MM and sub-MM wave $(\mathrm{THz})$ polarizers are needed in astrophysics (detection of polarization of Cosmic Microwave Background at the level of about $-80 \mathrm{~dB}$ ), polarization interferometry, remote sensing, etc.

Polarizing efficiency of grids decreases with increasing the frequency. A significant improvement can be achieved by using multi-layer structures (photonic devices) of subwavelength period of each grid, though of resonant inter-layer spacing [1]. The latter would improve the polarizer performance in the $\mathrm{THz}$ band when using rather coarse grids which are mechanically strong, inexpensive, and easy to produce and operate.

Though many devices benefit from utilizing multi-grid structures (see, e.g., [2]), there is, it seems, only one report [3] that suggests to exploit multiplication of extinction ratio of two parallel polarizers in tandem for actually improving the polarizers. The authors, however, based their conclusions on Mueller matrices of abstract polarizers that do not account for self-consistent solution of the electromagnetic problem and, as a result, missed a range of frequency-selective properties.

We present and compare the results of theoretical and experimental investigations of dual-layer grid polarizers designed for MM and sub-MM wave bands. Both the theory and experiment confirm a resonant quadratic growth of polarizing efficiency of dual-layer grid polarizers that occurs, depending on the operation mode, either at the discrete "spike" frequencies (when considering reflected waves) or in the broad frequency bands (when transmission waves are considered). As an example, polarizing efficiency of up to $60 \mathrm{~dB}$ at the frequencies of about $100 \mathrm{GHz}$ could be obtained with rather robust design (Figs. 1,2) that utilizes wire grids of relatively thick and sufficiently strong stainless-steel wire (e.g., of thickness $w=0.08 \mathrm{~mm}$ ).

Theoretical analysis and computer simulations of dual-layer grid polarizers have been made earlier [1] by utilizing either Wainshtein's approximation for analytical description of subwavelength grids in free space [4] or regularization technique for numerical modeling of microstrip gratings on thin-film substrates [5] (in all cases, the grid period $p$ is small as compared to the radiation wavelength $\lambda$ ).

Quadratic enhancement of polarization in transmission is achieved due to repeated reflection of the wave of rejected polarization (the one with the electric field parallel to grid wires) from both gratings in the assembly, thus, reducing unwanted polarization in the transmitted wave. The latter occurs at all but the resonant frequencies (those where inter-grid spacing $d$ satisfy the condition $d=n \lambda / 2$ ). At the resonant conditions, however, polarization in transmission drops significantly, since a dual-layer assembly in this case becomes transparent for all waves regardless polarizations.

In reflection, on the contrary, enhanced polarization appears only at the discrete "spike" frequencies that correspond to the quarter-wavelength inter-grid spacing in the dual-layer assembly. In this case, it is due to destructive interference of relatively weak reflected waves of unwanted orthogonal polarization (the one with the electric field orthogonal to grid wires) that enhancement of polarization occurs in reflection.

A few sets of polarizer-analyzer pairs of dual-layer wire-grid polarizers of different aperture diameters ( $D=90 \mathrm{~mm}$ and $D=170 \mathrm{~mm}$ ) have been fabricated for quasi-optical electromagnetic testing in the frequency band of $f=75-110 \mathrm{GHz}$ (Fig. 1). Each polarizer has been made as an assembly of two parallel wire grids stretched over the frame of appropriate aperture and thickness. The grid parameters are chosen to be as follows: $w=0.08 \mathrm{~mm}, p=0.27 \mathrm{~mm}, d=0.80 \mathrm{~mm}, D=90 \mathrm{~mm}$ for the polarizers of one kind (small-aperture polarizers), and $w=0.09 \mathrm{~mm}, p=0.33 \mathrm{~mm}, d=1.00 \mathrm{~mm}, D=170 \mathrm{~mm}$ for the polarizers of another kind (large-aperture polarizers). 


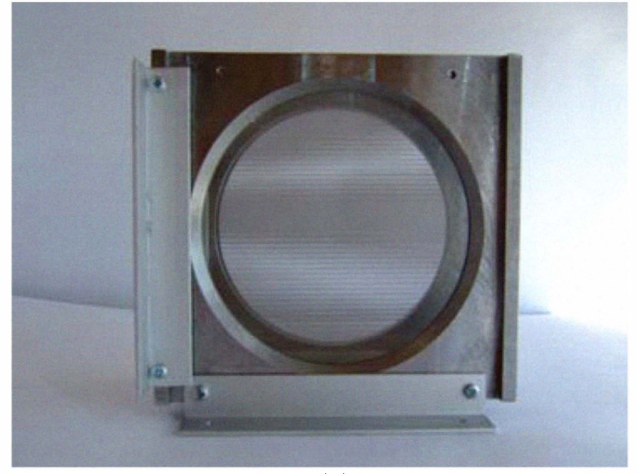

(a)

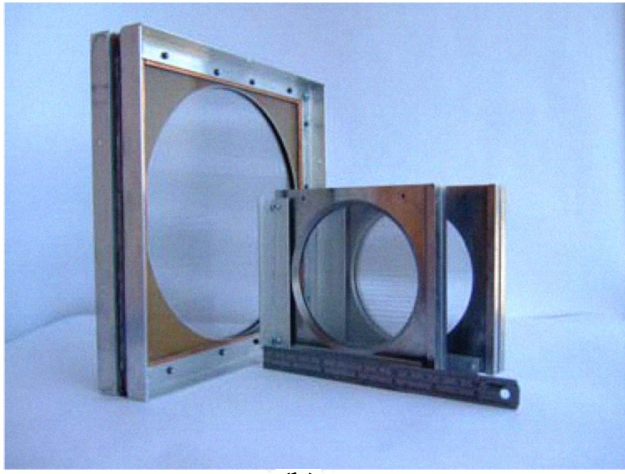

(b)

Figure 1. (a) Two dual-layer grid polarizers in tandem $(w=0.08 \mathrm{~mm}, p=0.27 \mathrm{~mm}, d=0.8 \mathrm{~mm}, \mathrm{D}=90 \mathrm{~mm})$ with a Moire pattern visible due to four grids overlapping in the aperture area (a second polarizer is slightly tilted with respect to the line-of-sight). (b) Moire patterns in the apertures of dual-layer polarizers show sufficient quality of grids. A more complicated second-order pattern in the overlapping apertures of small polarizers $(D=90 \mathrm{~mm})$ is due to the interplay between four identical grids.

Two grids in each assembly were made to be sufficiently planar and parallel, with non-planarity and nonparallelism of grid planes less than plus-minus $0.02 \mathrm{~mm}$ over the aperture area. The requirement on the uncertainty of inter-layer spacing allows us to keep polarization ratio at the "spike" frequencies in reflection at the same level of $60 \mathrm{~dB}$ as in transmission. Then, spectral curves of polarization extinction ratio at the plane wave incidence with account of this imperfection look the same as the curves computed for the perfect structure at the Gaussian beam incidence (brown curves in Fig. 2). Similarly, misalignment of wires of two grids is made to be better than 3 arcminutes that corresponds to misfit of wires of different grids by about $w=0.08 \mathrm{~mm}$ at one end of the grid pair as compared to the other end (assuming the grid aperture diameter $D=90 \mathrm{~mm}$ ).

Quality of grids can be verified by observing the Moire patterns that appear due to the interplay between four identical grids when overlapping the apertures of two polarizers in tandem as shown in Fig. 1, a and b. Wire-grid polarizers of this kind are supposed to be well suited for low-loss high-power applications, yet providing high polarizing performance (at the level of $-60 \mathrm{~dB}$ of polarization extinction ratio) in the frequency band around $f=100 \mathrm{GHz}$ where electromagnetic testing has been made.

Dual-layer grid polarizers made of the wire grids described above have been tested in the frequency band of $f=75-110 \mathrm{GHz}$ by free-space beam propagation method using a Vector-Network-Analyzer (VNA) highfrequency test facility operating at the NUI Maynooth (Ireland).

Requirements for high-frequency testing of enhanced polarizers are demanding. In addition to precise positioning of polarizers in cross-orientation with accuracy better than 3 arcminutes for maintaining spurious signals below $-60 \mathrm{~dB}$, detection of signals of ultra-low level is needed. Other significant requirement are the need to have a well-collimated beam, narrow angular patterns of detectors $\mathrm{R}$ and $\mathrm{T}$ for the reflected and transmitted waves, respectively, and sufficiently large aperture diameters of polarizers, so that no edge effects could contaminate the measurements at so low level of signals as expected in this experiment.

In order to satisfy all the requirements, a special set of quasi-optical focusing elements (ellipsoidal reflector mirrors with extra dual-layer polarizers at both the source and detector sites) had to be applied that appeared to be a crucial development, especially, when measuring reflected signals from the polarizer by the detector $\mathrm{R}$ in the case of oblique beam incidence.

The results of high-frequency electromagnetic testing of dual-layer grid polarizers in both transmission and reflection configurations are summarized in Fig. 2, a. The results are presented for the polarizers of small diameter $(D=90 \mathrm{~mm}, w=0.08 \mathrm{~mm}, p=0.27 \mathrm{~mm}$ ) at oblique wave incidence $(\vartheta \approx 20$ degrees, $d=0.80 \mathrm{~mm})$.

Curves 1 to 4 in Fig. 2, a, show the power in $\mathrm{dB}$ of the signal received by the relevant detector $(\mathrm{R}$ or $\mathrm{T})$ versus frequency in $\mathrm{GHz}$ in the following configurations, respectively:

1 - signal going through in co-polar grid position of the polarizer P2 (horizontal grid parallel to $\mathrm{H}$-field, large signal going through),

2 - signal reflected in cross-polar grid position of the polarizer P2 (vertical grid parallel to E-field, large signal reflected),

3 - signal reflected in co-polar grid position of the polarizer P2 (horizontal grid parallel to H-field, small signal reflected), 
4 - signal going through in cross-polar grid position of the polarizer P2 (vertical grid parallel to E-field, small signal going through).

All the curves represent the signals normalized with respect to the calibration signal received by detector $\mathrm{T}$ when only one polarizer P1 is present. For the comparison, smooth curves (light-blue and brown ones) show the simulation results obtained for the polarizers of this kind at the oblique beam incidence.

The measurements show that, for the dual-layer grid polarizer of this design, polarization extinction ratio is achieved at the level of $-65 \mathrm{~dB}$ to $-55 \mathrm{~dB}$ in the frequency band of $f=75-110 \mathrm{GHz}$ in transmission and $-60 \mathrm{~dB}$ at the "spike" frequency $f=95 \mathrm{GHz}$ in reflection (at the beam incidence angle of 20 degrees, where both the "spike" frequency and the relevant incidence angle are defined by the grid interlayer spacing).

Polarization ratios that can be achieved, according to simulations, with polarizers of this kind and of the finer grids in the broader frequency range are shown in Figs. 2, b and c, respectively.

Acknowledgement. Effort sponsored by the Air Force Office of Scientific Research, Air Force Material Command, USAF, under grant number FA8655-08-1-3049. The U.S. Government is authorized to reproduce and distribute reprints for Govemment purpose notwithstanding any copyright notation thereon.

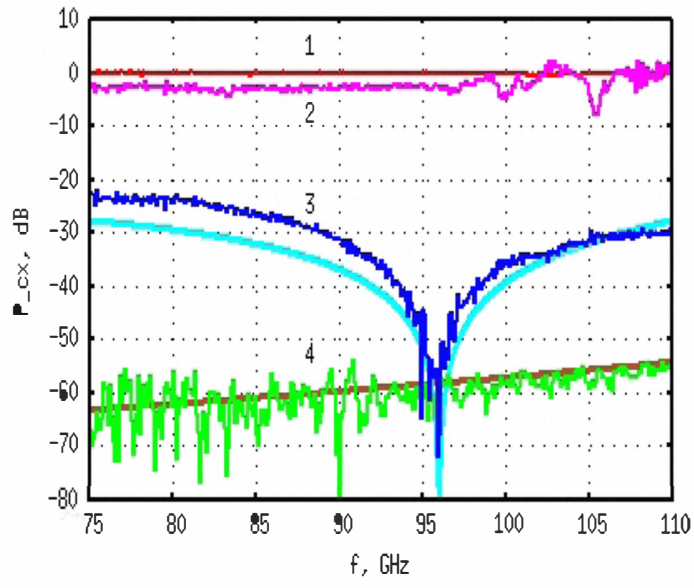

(a) (b)

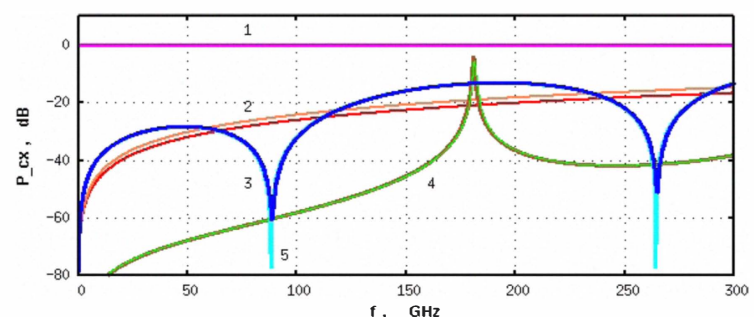

(c)

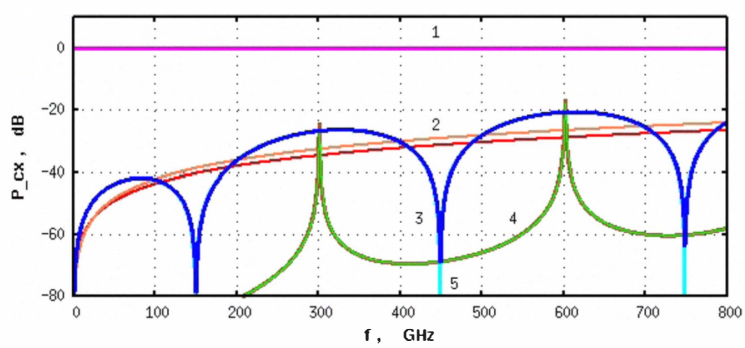

Figure 2. (a) Measurements made with a VNA facility at NUI Maynooth in reflection (curves 2, 3) and transmission (curves 1, 4) in co- (curves 2,4) and cross-orientation (curves 1,3) of polarizer grids with respect to the incident E-field (oblique incidence, $\theta \sim 20 \mathrm{deg}$; smooth curves represent simulation results). (b) Simulations that correspond to the experiment above when extended into the higher frequency band. (c) Simulations for the fine-grid polarizer $(w=0.01 \mathrm{~mm}, \mathrm{p}=0.033 \mathrm{~mm}, \mathrm{~d}=0.50 \mathrm{~mm})$ at the normal beam incidence in the frequency band up to $\mathrm{f}=800 \mathrm{GHz}$.

\section{References}

1. V. B. Yurchenko and E. V. Yurchenko, "Dual-Layer Frequency-Selective Subwavelength-Grid Polarizers for THz Applications," in Proc. 6th Intl. Kharkov Symposium on Physics and Engineering of Microwaves, Millimeter and Submillimeter Waves (MSMW-07), 25-30 June 2007, Kharkov, Ukraine, pp. 222-224, 2007

2. M.S. Yanovskiy, B.N. Knyaz'kov, E.M. Kuleshov, "Polarizer Attenuators for Quasi-Optical Track," Izv. vuzov-Radioelekronika, vol.17, no.9, pp.49-54, 1974 [in Russ.]

3. J. H. W. G. den Boer et al., "Improved polarizer in the infrared: Two wire-grid polarizers in tandem," Opt. Lett., vol.20, no.7, pp.800-802, 1995

4. L. A. Wainshtein, "To electrodynamic theory of gratings. Part I, II", in Elektronika Bol'shikh Moshchnosrei, Vol.2, P.L. Kapitsa and L.A. Wainshtein, Eds., Moscow, 1963, pp.26-73 [Engl. transl. in High-Power Electronics. Oxford: Pergamon, 1966, pp. 14-48].

5. V. B. Yurchenko, J. A. Murphy, J. Barton, J. Verheggen, and K. Rodgers, "Dual-Layer FrequencySelective Grid Polarizers on Thin-Film Substrates for THz Applications", in Proc. EuMW 2008: 38th European Microwave Conference 2008 (EuMC-2008), 28-31 October, 2008, Amsterdam, The Netherlands, pp. 10-14 - 10-17, 2008 